\title{
Der neue Spinozismus und das Verhältnis von deskriptiver und revisionärer Metaphysik
}

\author{
Renz, Ursula
}

\begin{abstract}
Relying on the assumption that Spinoza makes a double use of the principle of sufficient reason, Michael Della Rocca has defended a reconstruction of Spinoza's approach as a metaphysical outlook according to which all particulars vanish in the only and one divine substance. This implies nothing less than a radical attempt to suggest a new and completely revisionary form of metaphysics. After a short discussion of Strawson's distinction between revisionary and descriptive metaphysics and an exposition of the basic principles of Della Rocca's interpretation, I critically assess his attack of the use of intuitions in analytic philosophy. After discussing the extent to which the first book of Spinoza's Ethics is appropriately described as a revisionary project, I conclude with an argument for the necessity of both descriptive and revisionary elements in metaphysics
\end{abstract}

DOI: https://doi.org/10.1515/dzph-2015-0029

Posted at the Zurich Open Repository and Archive, University of Zurich ZORA URL: https://doi.org/10.5167/uzh-136673

Journal Article

Published Version

Originally published at:

Renz, Ursula (2015). Der neue Spinozismus und das Verhältnis von deskriptiver und revisionärer Metaphysik. Deutsche Zeitschrift für Philosophie, 63(3):476-496.

DOI: https://doi.org/10.1515/dzph-2015-0029 


\section{Ursula Renz*}

\section{Der neue Spinozismus und das Verhältnis von deskriptiver und revisionärer Metaphysik}

DOI 10.1515/dzph-2015-0029

Abstract: Relying on the assumption that Spinoza makes a double use of the principle of sufficient reason, Michael Della Rocca has defended a reconstruction of Spinoza's approach as a metaphysical outlook according to which all particulars vanish in the only and one divine substance. This implies nothing less than a radical attempt to suggest a new and completely revisionary form of metaphysics. After a short discussion of Strawson's distinction between revisionary and descriptive metaphysics and an exposition of the basic principles of Della Rocca's interpretation, I critically assess his attack of the use of intuitions in analytic philosophy. After discussing the extent to which the first book of Spinoza's Ethics is appropriately described as a revisionary project, I conclude with an argument for the necessity of both descriptive and revisionary elements in metaphysics.

Keywords: Baruch de Spinoza, individual, monism, principle of sufficient reason, revisionary metaphysics, descriptive metaphysics, P. F. Strawson, methods in the history of philosophy, meta-philosophy.

Nicht nur tote, sondern auch prima facie nicht lebensfähige Hunde können auferstehen. So jedenfalls ließe sich jenes Schauspiel zusammenfassen, das sich derzeit auf der Bühne der amerikanischen Philosophiegeschichte abspielt. Nachdem Spinoza während Jahrzehnten im Schatten von Hume, Descartes und Aristoteles stand und sein Ansatz oft dafür kritisiert wurde, dass seine Sichtweise auf die Welt und die sich in ihr befindenden Dinge unmöglich mit unseren alltäglichen Begriffen und Intuitionen in Übereinstimmung gebracht werden könnte, ${ }^{1}$ hat sich in den letzten Jahren der Wind gedreht. Spinozas Philosophie und insbesondere seine Metaphysik werden in den Dienst einer Bewegung genommen, die man auch als Rehabilitierung revisionärer Metaphysik beschreiben kann. Inspira-

1 Vgl. dafür exemplarisch die mittlerweile klassische Interpretation von Curley (1969) sowie den Kommentar von Bennett (1984).

*Kontakt: Univ.-Prof. Dr. Ursula Renz: Alpen-Adria-Universität Klagenfurt, Institut für Philosophie, Universitätsstr.65-67, 9020 Klagenfurt, Österreich; ursula.renz@aau.at. 
tor dieser Bewegung ist Michael Della Rocca, der Spinoza nicht nur einen radikalen, in einen Idealismus übergehenden Rationalismus zuschreibt, ${ }^{2}$ sondern in seiner Monografie Spinoza sämtliche Doktrinen des Titelhelden, angefangen von seiner Metaphysik und Philosophie des Geistes über seine Psychologie und Ethik bis hin zu seiner Rechts- und Staatsphilosophie und Bibelhermeneutik auf der Basis der Annahme rekonstruiert, dass dieser eine radikale Version des Prinzips des zureichenden Grundes (= PSR für „principle of sufficient reason“) vertreten und verwendet habe. ${ }^{3}$

Diese radikal-rationalistische Interpretation ist nicht unwidersprochen geblieben, ${ }^{4}$ dennoch ist sie eine Erfolgsgeschichte. Sie hat die Spinoza-Forschung stark inspiriert, und dies selbst dort, wo Interpreten andere Wege gehen. ${ }^{5}$ Darüber hinaus wird Della Roccas Spinozismus zunehmend auch außerhalb der Philosophiegeschichte zur Kenntnis genommen, so etwa in der Diskussion über einen metaphysischen Monismus ${ }^{6}$ oder im Blick auf die Frage des „metaphysischen Groundings“. ${ }^{7}$ Im Rahmen der in Harvard gehaltenen Whitehead Lectures im Frühling 2014 hat Della Rocca ferner seinen - wie er es nun nennt - „Parmenideischen Aufstieg“ („Parmenidean Ascent“) unabhängig von Spinoza verteidigt. Dabei wendete er ehemals in der Rekonstruktion von Spinozas Metaphysik entwickelte Überlegungen zur numerischen Differenz von Dingen auch auf Begriffe wie „Handlung“ und „Wissen“ an. Dabei gelangte er zu einer Position, die er jenseits selbst eines Monismus ansiedelt und derzufolge es metaphysisch gesprochen nicht mehrere, voneinander verschiedene Handlungen oder Wissensbestände geben kann, sondern nur „Aktivität“ oder „Wissen“, wovon, was wir im Alltag als Handlungen oder Wissensbestände ansprechen, nur Teile oder Manifestationen wäre. ${ }^{8}$

Dies ist ein kühner Gedanke, der von der Überzeugung getragen ist, dass man, will man dem PSR Rechnung tragen, in der Explikation von Begriffen der

2 Vgl. Della Rocca (2012a).

3 Vgl. ders. (2008).

4 Vgl. dazu etwa Laerke (2011), 446-62; Melamed (2012); Newlands (2010), 489; ders. (2012), 46-48; sowie meine eigenen Ausführungen in Renz (2010), 12-13.

5 Vgl. dazu Laerke (2014), der zur Darstellung dieser Diskussion auch auf Topoi der Konstellationsforschung zurückgreift.

6 Vgl. dazu auch den Beitrag von Dominik Perler zu diesem Schwerpunkt (Perler 2015).

7 Siehe dazu Della Rocca (2012b).

8 Ich danke Michael Della Rocca dafür, dass er mir die Manuskripte dieser Vorlesungen zur Verfügung gestellt hat. Zu erwähnen ist, dass er sich in den Whitehead Lectures selbst nur auf Parmenides und nicht auf Spinoza bezieht. Dennoch ist die Entwicklung dieser extremen und kontraintuitiven Position ohne die früheren Arbeiten zu Spinoza kaum denkbar. 
Frage nach ihrer Begründbarkeit den Vorrang vor jener nach dem deskriptiven Gehalt oder analytischen Potential einräumen muss, und dies unabhängig davon, ob man es gerade mit dem abstrakten Vokabular der großen Metaphysiker oder mit Alltagsbegriffen wie ,Handlung‘ oder ,Wissen` zu tun hat. Della Rocca spricht sich somit für eine rein revisionäre Metaphysik unter der Leitung jenes radikalen Rationalismus aus, wie er ihn zuvor im Rahmen seiner Rekonstruktion der Philosophie Spinozas entwickelt hat. ${ }^{9}$

In der Folge soll daher die Frage nach der Aktualität Spinozas zum Anlass genommen werden, das Verhältnis von revisionärer und deskriptiver Metaphysik kritisch zu reflektieren. Dazu möchte ich in einem ersten Schritt auf jene Erörterungen eingehen, mit denen Peter F. Strawson seinerzeit diese Unterscheidung zweier idealtypischer Arten der Metaphysik eingeführt hat. In einem zweiten Schritt werde ich dann kurz darlegen, wie Della Rocca aus einer rein metaphilosophischen Überlegung heraus Spinozas Substanzmonismus in seiner radikalsten und zugleich kontraintuitivsten Lesart rekonstruiert und verteidigt. Es wird sich zeigen, dass in seiner Interpretation Spinozas Metaphysik nicht nur in wesentlichen Zügen von unseren alltäglichen Intuitionen abweicht, sondern letzteren gar kein argumentatives Gewicht mehr zugestanden wird. Vor diesem Hintergrund möchte ich in einem dritten Schritt auf Della Roccas Kritik am Umgang der analytischen Philosophie mit unseren Intuitionen zu sprechen kommen, die er, die kontraintuitiven Resultate seiner Rekonstruktion rechtfertigend, im Zuge einer Stellungnahme zu methodologischen Fragen der Philosophiegeschichte vorgenommen hat. Erst im vierten Teil werde ich zu einer Kritik an Della Roccas Spinoza-Interpretation und deren Umgang mit dem Prinzip des zureichenden Grundes übergehen, bevor ich im fünften Teil auf einer grundsätzlichen Ebene das Verhältnis von Deskription und Revision in der Metaphysik reflektiere.

\section{1}

In der Einleitung zu Individuals rechtfertigt Strawson sein Ziel, die invarianten Strukturen unseres alltäglichen Begriffssystems zu explizieren, bekanntlich damit, dass er sein Unterfangen als einen „Versuch in deskriptiver Metaphysik“ deklariert und vom als revisionär apostrophierten Projekt gewisser Klassiker abgrenzt. Den damit stipulierten Gegensatz von deskriptiver vs. revisionärer Metaphysik erläutert er mit folgenden Worten:

9 Von zentraler Bedeutung sind Della Rocca (2003) und ders. (2012a). 


\begin{abstract}
Deskriptive Metaphysik ist zufrieden damit, die aktuelle Struktur unseres Denkens über die Welt zu beschreiben; revisionäre Metaphysik ist damit befasst, eine bessere Struktur hervorzubringen. Die Produktion von revisionären Metaphysiken ist von bleibendem Interesse, und das nicht nur, weil sie Schlüsselepisoden in der Geschichte des Denkens darstellen. Wegen der Artikulation und Intensität ihrer partiellen [und parteiischen] Visionen sind sie nicht nur an sich bewundernswert, sondern von einem bleibenden philosophischen Nutzen. Dieses letztere Verdienst kann ihnen aber nur zugeschrieben werden, weil es eine andere Metaphysik gibt, die keinerlei Rechtfertigung bedarf außer jener, welche Forschung im Allgemeinen betrifft. Revisionäre Metaphysik steht im Dienst der deskriptiven Metaphysik. Vielleicht hat zwar kein Metaphysiker - und zwar weder der Intention nach, noch im Resultat - je das eine oder andere [Programm] vollständig realisiert. Dennoch können wir grob unterscheiden: Descartes, Leibniz, Berkeley sind revisionäre, Aristoteles und Kant deskriptive Metaphysiker. ${ }^{10}$
\end{abstract}

Diese Gegenüberstellung zweier idealtypischer Arten von Metaphysik ist auf Anhieb ziemlich einleuchtend, zieht sie doch eine klare und, so scheint es, begründete Linie zwischen philosophischen Ansätzen, in denen wir unsere üblichen Ansichten über die Welt säuberlich auseinandergefaltet wiederfinden, und solchen, in die wir uns in einem eigentlichen Sinn des Wortes hineindenken müssen, um mit ihnen etwas anfangen zu können. Bei einer genaueren Betrachtung der Bemerkungen, mit denen Strawson diese Unterscheidung einführt, treten indes einige Merkwürdigkeiten zutage.

Erstens ist frappant, dass zwar von einer Mehrzahl revisionärer Metaphysiken, aber nur von einer deskriptiven Metaphysik die Rede ist. Vor dem Hintergrund seines Verständnisses von deskriptiver Metaphysik ist das zwar nicht erstaunlich: denn Strawson geht davon aus, dass das menschliche Denken in seinem Kern unverändert bleibt und von historischen Einflüssen nicht weiter tangiert wird. ${ }^{11}$ Ferner nimmt er an, dass das menschliche Denken einen systemischen Charakter hat. Diese Annahmen legen nahe, dass es nur eine einzige vollständige gültige Explikation der unserem alltäglichen Begriffssystem zugrunde liegenden Regeln geben kann. ${ }^{12}$ Demgegenüber sind Versuche, dieses Regelwerk $\mathrm{zu}$ verändern, in so großer Zahl möglich, wie man sich kreative Ideen zu einer Verbesserung unseres Alltagsverstandes ausdenken kann. Dass der Vielzahl von revisionären Metaphysiken nur eine einzige deskriptive Metaphysik gegenübersteht, ist somit ein integraler Bestandteil von Strawsons Programm. Dennoch

10 Vgl. Strawson (1971), 9 (Übers. U. R.).

11 Vgl. ebd., 10.

12 Vgl. dazu die Ausführungen zum Bild der spanischen Grammatik, anhand dessen Strawson in Analysis and Metaphysics sein Verständnis philosophischer Begriffsklärung erläutert (ders. 1992, 5-6). 
kann man die kritische Frage aufwerfen, ob Strawson nicht allzu großzügig darüber hinweg gesehen hat, dass es historisch betrachtet ebenfalls mehrere Versuche, die Grundstrukturen unseres Begriffssystems zu explizieren, gegeben hat.

Zweitens sticht ins Auge, dass Strawsons Darstellung zufolge die historischen Versuche einer Revision unseres Begriffsystems nicht bloß de facto nützlich für das Projekt einer deskriptiven Metaphysik sind. Überdies wird kategorisch festgehalten, dass ein solcher Nutzen revisionären Ansätzen überhaupt nur attestiert werden könne, weil es eine andere Art, Metaphysik zu betreiben, gebe, die keiner spezifischen Rechtfertigung bedürfe. Demgegenüber wird die umgekehrte Möglichkeit, dass die Analysen der deskriptiven Metaphysik einer Revision der Struktur unseres Denkens zuarbeiten könnten, gar nicht in Betracht gezogen. Damit setzt Strawson zwischen den beiden Metaphysiktypen ein dezidiert asymmetrisches Verhältnis an.

Schließlich ist drittens darauf hinzuweisen, dass Strawson keine Begründung für seine Auffassung gibt, dass Descartes, Berkeley und Leibniz revisionäre, Aristoteles und Kant hingegen deskriptive Metaphysiker gewesen seien. Strawson würde hier wohl entgegnen, dass sich diese Zuweisungen von der Sache her von selbst verstünden. Die Ansätze von Descartes, Berkeley und Leibniz entsprächen nun einmal nicht unseren alltäglichen Auffassungen, während Aristoteles und Kant diese einigermaßen richtig wiedergäben. In der Tat finden sich in Individuals etliche Passagen, in denen - wenn auch mehr oder weniger indirekt Descartes' Dualismus, Berkeleys psychologischer Idealismus und Leibniz' Monadenbegriff zurückgewiesen werden, während umgekehrt die kantische und v. a. aristotelische Begrifflichkeit öfters als mit unserem Begriffssystem in Einklang stehend auftreten. Ob Strawson damit den als revisionär taxierten Ansätzen von Descartes, Leibniz und Berkeley gerecht wird, muss hier offen bleiben. An diesem Umgang mit philosophiehistorischen Positionen wird indes deutlich, dass seiner Gegenüberstellung zweier Metaphysik-Typen die Versuchung zu einem von Polemik nicht freien Konservativismus innewohnt.

Zusammenfassend können wir somit festhalten, dass die Unterscheidung zwischen deskriptiver und revisionärer Metaphysik mindestens in der Strawson'schen Fassung keine neutrale ist, sondern mit einer klaren Präferenz für eine beschreibende Metaphysik einhergeht. Für ihre Verwendung dieser Unterscheidung zur Rekonstruktion von philosophiehistorischen Positionen ist das allerdings kein Hindernis: denn obwohl es nicht immer leicht zu entscheiden ist, ob man es bei einer Position mit einem deskriptivem oder mit einem revisionären Ansatz zu tun hat und es überdies viele Mischformen gibt, ist Strawsons Unterscheidung für heuristische Zwecke sehr brauchbar. Für die zeitgenössische Metaphysik wird sie indes zur Hypothek. Wer heute von unserem alltäglichen Verständnis abweichende Positionen vertreten will, muss sich immer erst von 
einem seiner metaphysischen Überväter emanzipieren. Das ist nicht so leicht denn wer bekennt sich schon gerne offen dazu, dass seine Thesen in direktem Widerspruch zu unseren Intuitionen stehen?

\section{2}

Für die Entwicklung revisionärer Positionen kann es von Vorteil sein, wenn man sich an philosophiehistorischen Vorbildern orientieren kann. Schreibt man eine revisionäre Auffassung anderen zu, so ist man - vorerst - davon entbunden, sie selber zu vertreten. Dass dadurch viele Annahmen oft nur als Interpretationshypothesen zur Diskussion stehen, mag zwar unbefriedigend erscheinen. Der dadurch gewährte Aufschub ist allerdings nicht hoch genug zu veranschlagen, erlaubt er es einem doch, die Voraussetzungen und Folgen fraglicher Positionen zu klären, ohne dass man sich entscheiden müsste, wie man es mit ihnen im Letzten halten wollte. Dazu kommt, dass alte philosophische Texte oft fragmentarisch sind oder aufgrund der historischen Distanz enigmatisch anmuten. Dies lädt oft dazu ein, sie mittels ungewöhnlicher Auffassungen zu erläutern, was in systematischer Hinsicht enorm inspirierend sein kann. Schließlich kann der Philosophiehistoriker in der Rekonstruktion historischer Ansätze die systematischen Möglichkeiten alternativer Sichtweisen ausloten, ohne sich damit zu belasten, sie mit unseren alltäglichen Überzeugungen abzustimmen. Nicht von ungefähr entwickelt sich deshalb die Diskussion von prima facie ungewöhnlichen Ansätzen oft zu einer Art Versuchslabor, wo auch dezidiert revisionäre metaphysische Ansichten untersucht werden können.

Um ein solches Versuchslabor handelt es sich zweifelsohne bei der jüngeren Debatte über Spinozas Metaphysik. In den letzten Jahren sind zahlreiche Untersuchungen erschienen, die sich dadurch auszeichnen, dass sie Spinoza auch dort beim Wort nehmen, wo dies zu sehr ungewöhnlichen und oft auch kontraintuitiven Positionen führt. Zu nennen sind hier vor allem die Debatte über Spinozas mutmaßlichen Nezessitarismus ${ }^{13}$ sowie die Diskussion über die mit seinem Substanzmonismus einhergehende These, dass es sich bei den Einzeldingen nur um sogenannte Modi handle. ${ }^{14}$ An beiden Schauplätzen sind in jüngerer Zeit vermehrt Stimmen laut geworden, die sich für revisionär-metaphysische Lesarten stark machen.

13 Für eine nezessitaristische Interpretation argumentieren etwa Garrett (1991) und Perler (2006), dagegen sprechen sich Curley/Walski (1991) sowie Renz (2009) aus.

14 Vgl. zur Erläuterung dieser These auch Perler (2015). 
Unter diesen sticht insbesondere die radikal-rationalistische Interpretation hervor, die Della Rocca in den letzten Jahren entwickelt hat. Den Ausgangspunkt bildet ein Aufsatz von 2002, worin Spinozas Argumentation für die Festlegung auf einen Substanzmonismus anhand dreier Interpretationshypothesen rekonstruiert wird. ${ }^{15}$ Erstens geht Della Rocca davon aus, dass für Spinoza „erklären“ und „begreifen“ dasselbe seien. Wenn Spinoza sage, dass $x$ durch $y$ begriffen werde, dann meine er, dass $x$ durch $y$ erklärt werde, und zwar durchaus in einem strikt kausalen Sinne von Erklären. ${ }^{16}$ Diese Interpretationshypothese ist nicht neu und sie wird in gemäßigteren Lesarten auch von Kritikern weitgehend akzeptiert. ${ }^{17}$ Della Rocca verbindet damit aber zwei weitere Annahmen, auf deren Grundlage er seine Rekonstruktion weiter zuspitzt.

Wenn, so die zweite Interpretationshypothese, Spinoza in seiner Argumentation für den in Lehrsatz 4 des 1. Buchs der Ethik (=1p4) behaupteten Substanzmonismus von der in 1p5 vertretenen These Gebrauch mache, wonach es in der Natur keine Substanz von derselben Natur resp. denselben Attributen geben könne, dann stütze er sich eigentlich auf eine bestimmte Version des Prinzips der Identität des Ununterscheidbaren (= PII für „Principle of the Identity of Indiscernibles“). ${ }^{18}$ Diesem Prinzip zufolge hätten wir es bei zwei Gegenständen nur dann mit verschiedenen Entitäten zu tun, wenn es ein Merkmal gebe, das ihre Nicht-Identität erkläre. Auf den ersten Blick ist auch diese Interpretationshypothese nicht sehr aufsehenerregend. Tatsächlich sagt Spinoza in 1p4, auf welchen Lehrsatz er sich in 1p5 stützt, nämlich jenen, demzufolge sich zwei oder mehrere Dinge „entweder anhand einer Verschiedenheit der Attribute der Substanzen oder anhand einer Verschiedenheit der Affektionen dieser Substanzen“ unterscheiden. ${ }^{19}$ Für Della Rocca ist nun aber entscheidend, dass Spinozas Version des PII nicht einfach festlege, dass es stets ein Merkmal oder eine Eigenschaft geben müsse, das die Differenz zweier Dinge ausmache, sondern dieses Merkmal

15 Laerke (2014), 723, sieht den Ursprung von Della Roccas radikalem Rationalismus in dessen Buch von 1996, worin er die idealistische These vertreten habe, dass es nichts als die Repräsentationen von Dingen gebe. Indem Laerke bereits Della Roccas frühe Rekonstruktion von Spinozas Philosophie des Geistes idealistisch wendet, geht er allerdings weiter, als Della Rocca es 1996 explizit getan hat.

16 Vgl. Della Rocca (2002), 11-12.

17 Vgl. für solche exemplarisch Wilson (2001), 141-65, sowie Renz (2010), 59-62.

18 Abgekürzte Verweise beziehen sich auf die Ethik in der Übersetzung von W. Bartuschat (Spinoza 1999). Es werden die üblichen Abkürzungen verwendet (def = definitio, ax = axioma, $\mathrm{p}=$ propositio, $\mathrm{d}=$ demonstratio, $\mathrm{s}=$ scholium, $\mathrm{c}=$ corollarium, app = appendix).

19 Ebd., 11. 
überdies von der Art sein müsse, dass es diese Differenz erkläre. ${ }^{20}$ Damit spricht Della Rocca Spinoza ab, dass sich Dinge durch nackte Tatsachen, wie es nach gängiger Auffassung etwa Zeit oder Ort der Existenz von Einzeldingen sind, von anderen unterscheiden: ${ }^{21}$ denn solche Bestimmungen erlauben es zwar, zwei Dinge als voneinander verschiedene zu beschreiben und zu erfassen, nicht aber, sie als voneinander verschieden zu erklären.

Della Roccas Spinoza zeichnet sich also durch eine extrem rationalistische Lesart des PII aus, derzufolge Unterschiede nur Bestand haben, wo sie in einem strikten Sinne des Wortes erklärt werden können. Doch wie lässt sich diese Interpretation rechtfertigen? An dieser Stelle bringt Della Rocca eine dritte Interpretationshypothese ins Spiel, die später für seine Entwicklung eines eigenen Ansatzes entscheidend sein wird. Seiner Auffassung nach verteidige Spinoza seine extrem rationalistische Lesart des PII mit einem Verweis auf ein anderes rationalistisches Prinzip, nämlich das Prinzip des zureichenden Grundes, dem Della Rocca, wie eingangs bereits angedeutet, mit der Abkürzung PSR seinen eigenen Stempel aufdrückt. Dieses Prinzip verlange, dass sämtliche Tatsachen erklärt werden können müssten, und dies gelte selbstverständlich auch für die Nicht-Identität von Dingen oder Eigenschaften. ${ }^{22}$ Della Rocca zufolge ist also das PII, auf das Spinoza in der Herleitung seines Substanzmonismus rekurriert, nur ein Sonderfall des PSR. Dies wiederum legt den Schluss nahe, dass letztlich die ganze Metaphysik Spinozas von diesem rationalistischen Prinzip abhängig ist.

Auch diese Auffassung ist nicht neu, vielmehr wurde Spinoza schon in etlichen Kontexten eine Orientierung am PSR attestiert. ${ }^{23}$ Della Roccas Inspiration dürfte dabei eine Überlegung von Don Garrett gewesen sein, der vor geraumer Zeit gezeigt hat, dass Spinozas Argument für einen Substanzmonismus an entscheidenden Stellen durch Überlegungen ergänzt werden muss, die sich nur rechtfertigen lassen, wenn man annimmt, dass Spinoza das PSR akzeptiert hat. Anhand einer Rekonstruktion von 1ax3 hat Garrett zudem gezeigt, dass Spinoza das PSR

20 Della Rocca (2002), 14.

21 Es sei hier nur am Rande festgehalten, dass 1p4 diese Auslegung nicht deckt, denn dort werden als mögliche Unterscheidungsmerkmale zwischen Dingen auch die Affektionen der Attribute der Substanzen genannt, die etwa in Zeit- oder Ortsbestimmungen bestehen können. Dass diese für die Individuation von Substanzen nicht relevant sein können, zeigt Della Rocca im Aufsatz von 2002 klar; die Frage stellt sich aber, ob der in diesem Text erstmals artikulierte Zusammenhang von Unterscheiden und Erklären tatsächlich für die Individuation aller Dinge gilt. 22 Ebd.

23 Vgl. Laerke (2014), 722, der verschiedene Belege aus der jüngeren französischen und deutschen Spinoza-Forschung zusammenträgt. 
tatsächlich aktiv vertreten haben könnte. ${ }^{24}$ Anders als Garrett zieht indes Della Rocca, wie oben skizziert, das PSR auch dazu heran, um Spinoza eine extremrationalistische Lesart des PII zuzuschreiben. Dadurch gewinnt das PSR einen vereinheitlichenden Drive, der in der Folge Della Roccas gesamte Rekonstruktion von Spinozas Denken bestimmt. Dieser zufolge führt die Annahme des PSR nämlich dazu, dass im Zuge der Ethik etliche Differenzierungen, von denen wir im Alltag ausgehen, preisgegeben werden. Davon betroffen ist nicht nur die mit dem Substanzmonismus explizit fallengelassene Annahme, dass es eine Mehrzahl selbständig subsistierender Dinge gebe. Vielmehr lässt Della Roccas Spinoza aufgrund seiner Verpflichtung auf das PSR auch zahlreiche andere unserem Alltagsdenken zugrundeliegenden begrifflichen Unterscheidungen fallen, wie z. B. jene zwischen Möglichkeit und Notwendigkeit, zwischen mentalen Vermögen und ihren Akten, zwischen mir und anderen, zwischen Erkennen und Wollen oder zwischen Recht und Macht. Darüber, wie weit die Annahme, dass Spinoza all diese Differenzierungen preisgebe, stimmt, und wie weit sie, wenn sie stimmt, tatsächlich unter Rekurs auf Spinozas mutmaßlichen Gebrauch des PSR verteidigt werden kann, lässt sich streiten. Ich werde im vierten Abschnitt noch kurz darauf eingehen. Hier möchte ich nur festzuhalten, dass es Della Rocca mit der Anwendung des PSR auf Tatsachen der Nicht-Identität von Gegenständen gelingt, zahlreiche Thesen, die Spinoza im Zuge der Ethik diskutiert, auf eine einzige Denkfigur zu reduzieren.

Der letzte und kühnste Schritt steht uns allerdings noch bevor. Della Rocca zufolge lässt es Spinoza nicht dabei bewenden, begriffliche Differenzierungen, wie sie unserem Alltagsvokabular zugrunde liegen, zu eliminieren. Entscheidend sei überdies, dass Spinoza einen zweifachen Gebrauch des PSR mache. ${ }^{25}$ Was damit gemeint ist, lässt sich exemplarisch an der Unterscheidung von Erklärbarkeit und Ursache verdeutlichen. Wenn wir nach der Ursache für eine bestimmte Wirkung fragen und also eine Erklärung dafür verlangen, dass etwas

24 Vgl. Garrett (1979), 201-202. Das Axiom 1ax3, worauf sich Garrett stützt, lautet: „Aus einer gegebenen bestimmten Ursache erfolgt notwendigerweise eine Wirkung; und umgekehrt, wenn keine bestimmte Ursache gegeben ist, ist es unmöglich, dass eine Wirkung erfolgt“ (Spinoza 1999, 9). Garrett zufolge kann 1ax3 dann als Evidenz für Spinozas Annahme des PSF gelten, wenn man erstens den Begriff der Ursache bei Spinoza so versteht, dass $x$ dann eine Ursache für $y$ ist, wenn $x$ (mindestens partiell) den Grund für die Existenz oder Natur von $y$ liefert; und wenn man zweitens 1ax3 liberal liest, d. h., auf alle Tatsachen bezieht und nicht nur auf Wirkungen bzw. Tatsachen, sofern sie eine Ursache haben. Die zweite Annahme ist m. E. durch die Semantik von „effectus“ nicht gedeckt; vgl. dazu Goclenius (1980), 142-143. Dies schließt aber nicht aus, dass Garretts liberale Lesart von 1ax3 Spinozas Absichten entspricht.

25 Vgl. dafür Della Rocca (2008), 8. 
so und nicht anders herausgekommen ist, dann können wir zwei Grundhaltungen einnehmen. Entweder glauben wir, dass es für die beobachtete Wirkung tatsächlich Ursachen gibt, die wir im Prinzip auch erkennen können und die eine vollständige Kausalerklärung für den zur Diskussion stehenden Sachverhalt abgeben können; oder aber wir glauben, dass verborgene Kräfte am Werk sind, die von keiner Erklärung erfasst werden können, und dass es mithin von vornherein ausgeschlossen ist, dass der gesuchte Kausalzusammenhang vollständig erklärbar wäre. Wir haben also die Wahl zwischen einer rationalistischen und einer skeptischen bzw. mystischen Position. Der erste Gebrauch des PSR, von dem Della Rocca spricht, ist nun genau dort am Werk, wo wir uns auf eine rationalistische Haltung gegenüber Kausalverhältnissen verpflichten, wo wir also annehmen, dass unsere Frage nach den Ursachen für einen Sachverhalt grundsätzlich vollständig beantwortbar ist. Mit dieser Annahme setzen wir voraus, dass die Verursachung und die Erklärbarkeit des zu erklärenden Sachverhalts koextensiv sind; d. h., wir gehen davon aus, dass $x$ genau dann durch $y$ verursacht wird, wenn $x$ durch $y$ erklärt werden kann. Dieser Gebrauch des PSR ist weitgehend im Einklang mit unseren alltäglichen Intuitionen. Tatsächlich verhalten wir uns, wann immer wir davon ausgehen, dass Dinge vollständig aufgeklärt werden können, als gute Rationalisten.

Della Roccas Spinoza geht über diesen Alltags-Rationalismus noch hinaus. Wenn wir nach den Ursachen für eine Wirkung fragen, machen wir meist noch eine andere Voraussetzung, nämlich, dass Verursachung und Erklärbarkeit zwar koextensive Prädikate sind, aber nicht dasselbe meinen. Indem wir nach den Ursachen von bestimmten Sachverhalten fragen, unterstellen wir, dass es ein vom zu erklärenden Sachverhalt verschiedenes, diesen erklärendes Merkmal gebe, welches wir in unserer Erklärung nur mehr als solches erfassen müssten. Wir gehen mit anderen Worten davon aus, dass wir es mit zwei ontologisch verschiedenen Ebenen zu tun haben. Während wir mit der Rede von der Verursachung auf eine Relation zwischen extramentalen Dingen aus sind, machen wir mit Aussagen über die Erklärbarkeit von Dingen eine Behauptung über die mögliche Reichweite von Erkenntnis. ${ }^{26}$

26 Georg Brun hat die Frage aufgeworfen, inwiefern man hier von einem ontologischen Unterschied sprechen könne. Man kann sich natürlich fragen, ob man diesen Unterschied zwischen den genannten Ebenen „ontologisch“ nennen will, wenn wir es doch mit zwei koextensiven Prädikaten zu tun haben, oder ob es nicht angemessener wäre, von Denkweisen ein- und desselben Dings zu sprechen. Ich denke aber, dass mit diesem Vorschlag ein sehr großer Druck auf unseren Alltagsverstand ausgeübt würde, der seinerseits einer Rechtfertigung bedürfte. 
Der zweite Gebrauch des PSR, den Della Rocca Spinoza attestiert, tangiert genau diese Unterscheidung. Wenn wir, so seine Überlegung, das PSR wirklich ohne Einschränkung akzeptieren, dann müssen wir auch annehmen, dass es für die Nicht-Identität von Kausalität und Erklärbarkeit einen Grund gibt. Doch worin sollte dieser bestehen? Tatsächlich haben wir ja mit dem ersten Gebrauch bereits zugegeben, dass Kausalität und Erklärbarkeit koextensiv sind, und haben mithin den Kausalrelationen, nach denen wir fragen, bereits jeglichen über ihre Erklärbarkeit hinausgehenden Realitätsüberschuss abgesprochen. Was, außer der Tatsache, dass wir die Ursache, nach der wir fragen, noch nicht kennen, hindert uns daran, den zweiten Schritt zu machen und Kausalität und Erklärbarkeit nicht nur als koextensiv, sondern als ein und dieselbe Eigenschaft von Relationen anzusehen? Offensichtlich können wir keinen anderen Grund für unser Beharren auf der Unterscheidung zwischen Erklärbarkeit und Kausalität namhaft machen als unsere eigene Unwissenheit, und stehen somit vor der Wahl, auch die unserem Fragen selbst zugrunde liegende Unterscheidung preiszugeben und zuzugestehen, dass Kausalität Erklärbarkeit ist, oder aber die Gültigkeit des PSR an dieser Stelle einzuschränken.

Für Della Rocca ist klar, dass Spinoza auch an dieser Stelle am PSR festhält und die ontologische Differenz zwischen Erklärbarkeit und Kausalität preisgibt. Dies hat ziemlich verheerende Folgen: denn abgesehen davon, dass diese Reduktion von Kausalität auf Erklärbarkeit ziemlich kontraintuitiv anmutet, tangiert sie etwas, das eine zentrale Voraussetzung unseres Fragens nach Ursachen ist. Während also das PSR ein erstes Mal dort am Werk ist, wo wir nach Gründen für ein bestimmtes Phänomen fragen und diese Frage für beantwortbar halten, besteht der zweite Gebrauch des PSR in der Eliminierung der in unserem Fragen nach Gründen vorausgesetzten operativen Unterscheidungen. ${ }^{27}$

Zusammenfassend können wir somit festhalten, dass sich der radikale Rationalismus, den Della Rocca Spinoza zuschreibt, nicht darauf beschränkt, jenen intuitiv plausiblen Rationalismus, der unser Fragen nach Gründen bestimmt, freizulegen und von überflüssigem terminologischem Schnickschnack zu reinigen. Darüber hinausgehend nimmt Della Roccas Spinoza mithilfe seines zweifachen Gebrauchs des PSR auch eine ontologische Reduktion letztlich sämtlicher Phänomene auf ihre Erklärbarkeit vor. Damit legt Della Rocca seinen Spinoza nicht mehr nur auf einen radikalen Rationalismus, sondern auch auf einen idealistischen Monismus oder Pantheismus fest.

27 Dies ist meine und nicht Della Roccas Formulierung. Er selber spricht davon, dass Spinoza Phänomene durch den Begriff der Erklärung erkläre: „Spinoza’s next move is to explain the phenomenon [in question] in terms of explanation itself" (ebd., 30). 


\section{3}

Wie soeben skizziert, erweckt Della Rocca unter Rekurs auf die Annahme eines doppelten Gebrauchs des PSR das alte Bild des Spinozismus als radikaler Pantheismus, das dem historischen Spinoza seit jeher zum Vorwurf gemacht wurde, wieder zum Leben ${ }^{28}$ - doch tut er dies in einem anderen Geist als frühere Interpreten. Während jene dieses Bild zum Anlass nahmen, Spinozas Philosophie in Bausch und Bogen zu verwerfen, sieht Della Rocca darin seine Stärke. Wie kein anderer habe Spinoza mit dem Rationalismus Ernst gemacht und auch dort am PSR festgehalten, wo sich fast alle anderen Philosophen mit nackten Tatsachen zufrieden geben; und wie kein anderer sei Spinoza bereit gewesen, den Tribut, den das PSR fordere, auch wirklich zu bezahlen und wesentliche Elemente unseres Common Sense abzuschreiben.

Doch warum, so fragt sich, sollen wir eine so extreme Form des Rationalismus annehmen, wenn dies mit so hohen Kosten verbunden ist? Ist es wirklich eine intellektuelle Tugend, ohne jegliche Rücksicht auf unsere alltäglichen Intuitionen einem einzigen metaphilosophischen Prinzip zu folgen? Auf diese Art Einwand sind zwei Reaktionen denkbar. Zum einen kann man zeigen, warum man das fragliche Prinzip trotz der Kosten, die mit ihm verbunden sind, akzeptieren sollte. In seinen Spinoza-Interpretationen hat Della Rocca meist diesen Weg einschlagen. ${ }^{29}$ Zum anderen kann man aber auch zum Gegenangriff blasen und die Frage danach aufwerfen, ob denn das, was wir verlieren, wirklich so einen großen Verlust darstellt.

Letzteres hat Della Rocca kürzlich im Rahmen einer Stellungnahme zu methodologischen Fragen der Philosophiegeschichte getan. Zielscheibe seines Gegenangriffs ist, was er als Methode der Intuition (= MI) bezeichnet. Darunter subsumiert er - bewusst pauschalisierend - sämtliche philosophischen Ansätze, deren Systeme entweder auf unsere Intuitionen abstellen oder diese benützen, um die Prinzipien, auf denen ein System aufbaut, zu revidieren. Auch Ansätze, die sich der auf Nelson Goodman und John Rawls zurückgehenden Methode des reflexiven Überlegungsgleichgewichts (= MRE für „method of reflective equilibrium“) bedienen, operieren laut Della Rocca mit einer Variante der MI. ${ }^{30}$

Doch was ist problematisch an der Orientierung an Intuitionen? Della Rocca nennt drei Punkte. Erstens moniert er, dass den Vertretern der MI begründbare

28 Ganz explizit wird dies in ders. (2012a) getan.

29 Vgl. dazu insbesondere das Schlusskapitel von ders. (2008), wo argumentiert wird, dass wir, um den Gebrauch des PSR einzuschränken, eine willkürliche Linie zwischen Bereichen und Phänomenen, die dem PSR unterlägen, und solchen, die dies nicht tun, ziehen müssten.

30 Vgl. ders. (2013), 178 u. 182. 
Kriterien dafür fehlten, welche Intuitionen sie zum Ausgangspunkt ihres Nachdenkens machten. ${ }^{31}$ Deshalb generiere der Rückgriff auf Intuition unweigerlich Systeme, die sich durch einen willkürlichen Konservativismus auszeichneten. ${ }^{32}$ Zweitens sei problematisch, dass sich die MI mit unseren Überzeugungen über die Realität - und mithin mit psychologischen Tatsachen -, nicht aber mit der Realität selber befasse. Ironischerweise kämen daher jene Philosophen der Gegenwart, deren Stolz es sei, sich „direkt“ und ohne Umweg über die Auseinandersetzung mit historischen Positionen den Phänomenen selber zuzuwenden, nie bei diesen an, denn sie nähmen diese stets nur durch den Schleier ihrer Intuitionen wahr. ${ }^{33}$

Spätestens an dieser Stelle würden sich vermutlich die Vertreter der MRE zu Wort melden und darauf hinweisen, dass diese beiden Einwände nur solche Formen des Vorgehens träfen, bei denen unsere Intuitionen lediglich bestätigt und geklärt würden, ohne dass sie selber zum Gegenstand einer kritischen Überprüfung gemacht würden. Genau dies sei aber bei der MRE anders, gehe es doch dort auch darum, Intuitionen zu korrigieren oder aber zu rechtfertigen. ${ }^{34}$ Zudem handle es sich bei den Überzeugungen, von denen die MRE ausgehe, gar nicht zwingend um Intuitionen; den Ausgangspunkt könnten jegliche Auffassungen bilden, denen wir unmittelbar und ohne sie eigens herleiten zu können Glaubwürdigkeit beimessen. Das einzige, worauf die MRE abstelle, sei die Unterscheidung zwischen inhaltlichen Festlegungen einerseits und Elementen des Systems andererseits, die überdies beide im Zuge eines Prozesses der wechselseitigen Abstimmung revidiert würden. ${ }^{35}$

Genau an diesem Punkt setzt Della Roccas drittes Bedenken gegen die MI ein. Das flexible „Give-and-Take“ zwischen Intuitionen und Prinzipien - oder auch zwischen inhaltlichen Festlegungen und systemischen Elementen - lasse es offen, wo wir die Linie zwischen dem, was wir behalten, und dem, was wir verwerfen, ziehen müssten. Nun könne, wie Quines Kritik an der Unterscheidung von analytischen und synthetischen Sätzen gezeigt habe, prinzipiell alles Gegenstand einer Revision sein. Es gebe keine in der Sache liegende Grenze, die anzeige, wo wir unseren Revisionen Einhalt gebieten müssten. Welche unserer Intuitionen und Prinzipien wir behielten und welche wir dagegen verwürfen, sei

31 Hier ist die Darstellung von Della Rocca etwas oberflächlich. Nicht nur nehmen Vertreter der MRE zu diesem Problem durchaus explizit Stellung (vgl. schon Rawls 1979, 67), sondern es ist auch durchaus kontrovers, ob es solcher Kriterien bedarf. Ich danke an dieser Stelle Georg Brun für seine Hinweise.

32 Della Rocca (2013), 190.

33 Ebd., 191-193.

34 Siehe dazu wie für die folgenden Überlegungen exemplarisch Brun (2014), v. a. 245-249. 35 Vgl. ebd., 240. 
daher letztlich nicht das Resultat von begründeten Überlegungen, sondern von mehr oder weniger willkürlichen Entscheidungen.

Dieser Hinweis ist nicht völlig verkehrt. Er trifft die MRE allerdings nicht an ihrer Achillesferse, sondern münzt eher einen wesentlichen Punkt ihres Selbstverständnisses in Polemik um. Die Vertreter der MRE verstehen ihre Methode nämlich dezidiert als eine imperfekte prozedurale Form der Rechtfertigung, was das Eingeständnis einschließt, dass auch die eigenen Resultate nur von vorläufigem Wert sind. ${ }^{36}$ Ferner entbehrt die Entscheidung darüber, welche Überzeugungen zu behalten, welche dagegen zu verwerfen sind, zwar einer letztbegründeten Absicherung, orientiert sich aber durchaus an Kriterien. Solche sind - neben der Balance, in die Festlegungen und Systemelemente durch den Reflexionsprozess gebracht werden, neben der schon genannten initialen Glaubwürdigkeit von Festlegungen und dem Respekt, den wir früher für wahr gehaltenen Festlegungen schulden insbesondere gewisse Desiderata an die im Einzelfall $\mathrm{zu}$ entwickelnde Theorie. Diese setzen sich einerseits aus allgemein-epistemischen Tugenden wie Einfachheit oder begrifflicher Klarheit, andererseits aus den spezifischen Bedürfnissen zusammen, denen wir mit der jeweiligen Theorie Rechnung zu tragen suchen. ${ }^{37}$

Nun zeigt dieser letzte Punkt indirekt auch, dass Della Rocca mit seiner Kritik am Umgang mit Intuitionen in der analytischen Metaphysik einen wunden Punkt getroffen hat. Tatsächlich stellt sich die Frage, ob diese Disziplin unseren Bedürfnissen, die wir an metaphysische Theorien haben, gerecht wird. Eine Gerechtigkeitstheorie, die uns nicht in der Lage versetzt, Richtlinien für gerechtes Handeln und gesetzliche Regulierungen zu formulieren, verfehlt ihren Zweck, auch wenn sie intuitiv einleuchtend und in sich kohärent ist. Ähnlich, so könnte man Della Roccas Einwände auf den Punkt bringen, verfehle eine Metaphysik, die uns in wunderbarer Weise vorführt und erklärt, was wir immer schon über ein Phänomen denken, ihren Zweck: denn weil sie sich nicht direkt mit der Realität auseinandersetze, sei sie gar nicht in der Lage, uns zu erklären, worin die Realität (eines fraglichen Phänomens) bestehe.

Womöglich mag das manche nicht wirklich befriedigen. So kann man sich beispielsweise fragen, was es denn heiße, sich mit der Realität (eines Phänomens) auseinanderzusetzen, und was mithin Metaphysik zu erklären habe. Auch lässt uns Della Rocca im Unklaren darüber, wie wir ohne eine Analyse unserer Intuitionen auf die Realität (eines Phänomens) zugreifen können. Gleichwohl ist es m. E. richtig, dass die analytische Metaphysik die Antwort auf die Frage nach den spezifischen Desiderata, derer sie mit ihren Theorien Rechnung trägt, ob der Auseinandersetzung mit unseren Intuitionen öfters schuldig geblieben ist.

36 Vgl. dazu exemplarisch Elgin (1996), 10-11.

37 Vgl. dazu Brun (2014), 240-242. 


\section{4}

Im vergangenen Abschnitt ist ein wichtiges Motiv für die Auseinandersetzung mit Spinoza angeklungen: Wo Philosophiegeschichte in einer systematischen Einstellung betrieben wird, da kann sie uns auf unvorhersehbare Art lehren, die Dinge in einer ganz anderen Weise zu sehen, als wir es gewohnt sind; und gerade ungewöhnliche Ansätze wie jener, mit dem uns die Ethik konfrontiert, sind oft hilfreich, wenn es darum geht, unsere Intuitionen, mit denen wir immer schon auf unsere Umgebung blicken, zu hinterfragen. Trotzdem ist es zweifelhaft, inwieweit Spinoza den Titel eines Retters vor dem Common Sense, den ihm Della Rocca im Zuge seiner Kritik an der zeitgenössischen Metaphysik verliehen hat, ${ }^{38}$ auch verdient. Wirft Spinoza in seiner Metaphysik wirklich so radikal sämtliche unserer alltäglichen Intuitionen über Bord, wie Della Rocca behauptet?

Diese Frage ist - wie viele Interpretationsfragen - nicht abschließend $\mathrm{zu}$ klären. Letztlich handelt es sich hier um eine hypothetische Frage: Zur Diskussion steht die Frage danach, wie radikal sich der Autor der Ethik dem die gegenwärtige Diskussion bestimmenden Trend zur deskriptiven Metaphysik widersetzt hätte. Darüber lässt der Text keine eindeutigen Schlüsse zu. Dennoch stehen wir als Philosophiehistoriker nicht mit leeren Händen da, sondern es gibt mehrere, an sich sehr einfache Methoden, die uns - klug angewendet - dabei unterstützen können, etwas verbindlichere Aussagen darüber zu treffen, wie ein Autor sich zu dieser Art hypothetischer Fragen verhalten hätte.

Die erste dieser Methoden besteht schlicht darin, zu schauen, was der Autor im Zuge der Rechtfertigung einer Theorie tut, statt einfach zu interpretieren, was er in ihr sagt. Dieser pragmatische Ansatz ist gerade im Zusammenhang mit metaphysischen Themen angebracht, denn diese sind stark mit unserer operativen Begrifflichkeit verwoben. Auch die Annahme, dass Spinoza in seiner Metaphysik vom PSR Gebrauch mache, resultiert aus diesem pragmatischen Ansatz. So wird das PSR in der Ethik weder wie andere Prinzipien axiomatisch eingeführt noch eigens gerechtfertigt, sondern nur im Zuge des zweiten Beweises von 1p11 erwähnt. ${ }^{39}$ Es fragt sich allerdings, ob Spinoza von diesem Prinzip auch den oben beschriebenen zweiten Gebrauch macht und eine ontologische Reduktion sämtlicher Phänomene auf ihre Erklärbarkeit vornimmt.

Dies kann mindestens hinsichtlich jener zwei Punkte bezweifelt werden, die für die Frage, ob Spinoza einen idealistischen Monismus vertreten habe, ausschlaggebend sind. Weder wird in der Ethik jegliche numerische Differenz

38 Siehe Della Rocca (2013), 185 u. 208.

39 Sowohl ders. (2008), 8, als auch Schütt (1985), 174, rekurrieren im Blick auf das PSR auf 1p11d2. 
zwischen den Einzeldingen preisgegeben, ${ }^{40}$ noch wird die Unterscheidung zwischen der Existenz und der Erklärbarkeit von Phänomenen aufgehoben. Zwar nimmt Spinoza an, dass alles, was sei, erklärbar sei, nicht aber, dass die Existenz von Dingen in ihrer Erklärbarkeit bestehe. ${ }^{41}$ Anzumerken bleibt, dass sich diese Aussage nur auf negative Evidenzen - also auf Feststellungen darüber, was der Text nicht tut - stützen kann.

Fruchtbarer ist daher eine andere Methode. Will man entscheiden, wie sich ein Autor zu einer Interpretationsfrage verhalten hätte, so ist es bisweilen lohnend, seine Position im historischen Kontext zu betrachten. Dies ist auch mit Blick auf die Frage danach möglich, ob Spinoza ein eher deskriptives oder eher revisionäres Programm verfolgt: denn was die Aufgabe der Metaphysik sei, war bekanntlich auch im 17. Jahrhundert Gegenstand von Debatten, setzte sich doch erst in jener Zeit die Trennung der natürlichen Theologie von der allgemeinen Metaphysik durch, wobei für letztere der Name ,Ontologie' geprägt wurde. ${ }^{42}$ Auch Spinoza nimmt in seiner Metaphysik auf diese Debatte Bezug. ${ }^{43}$

Doch wie genau verhält er sich dazu? Handelt es sich bei der im ersten Buch der Ethik entwickelten Metaphysik um eine natürliche Theologie oder um eine Ontologie? Obwohl der Titel dieses ersten Buches „De Deo“ dafür spricht, dass wir es mit einer natürlichen Theologie zu tun haben, deutet manches darauf hin, dass es sich bei diesem Text de facto um eine Ontologie handelt. So heißt etwa der

40 Siehe dazu auch Perler (2015). Vgl. für Spinozas Individuumsbegriff auch Renz (2010), 64-77, sowie für die numerische Differenz zwischen singulären Geistern ebd., 145-153 u. 189-196.

Michael Della Rocca hat gegen diese Aussage im Rahmen einer privaten Korrespondenz eingewendet, dass es sehr wohl Stellen in Spinozas Werk gebe, in denen die Realität der Einzeldinge explizit bezweifelt werde, und er verweist exemplarisch auf die Briefe 12, 19 u. 50. Ich möchte dies gar nicht in Abrede stellen, sondern ich stelle nur fest, dass Spinoza - und zwar obwohl er etwa im 12. Brief an Ludwig Meyer aus dem Jahr 1663 behauptet, dass „Maß, Zeit und Zahl nur Modi des Denkens“ seien - nie aufgehört hat, von mehreren Einzeldingen zu sprechen, auch nicht in der zweiten Hälfte des fünften Buchs der Ethik.

41 Eindeutigster Hinweis darauf, dass Spinoza diese Differenz nicht einebnen will, ist m. E. 2p8c; vgl. dazu auch Renz (2010), 160, sowie die noch weiter gehenden Erörterungen in dies. (i.E.).

42 Vgl. dazu Kremer (1984), 189-1990. Vorbereitet wurde diese Trennung natürlich schon früher. Gemäß Aristoteles handelt bekanntlich die erste Philosophie einmal vom Seienden als Seienden, dann von den ersten Ursachen und Prinzipien und schließlich von den abgetrennten und unbewegten Gegenständen. Diese konkurrierenden Bestimmungen führten im Mittelalter - namentlich bei Avicenna und Averoës sowie im Anschluss an Duns Scotus - zu Kontroversen darüber, was die primäre Aufgabe der Metaphysik sei. Die Trennung in zwei Disziplinen wird jedoch erst in der deutschen Schulphilosophie vorgenommen, und diese Entwicklung ist erst mit Christian Wolff abgeschlossen.

43 Diese These vertritt Robert Schnepf (1996) in seiner ebenso erhellenden wie historisch lehrreichen Rekonstruktion von Spinozas Metaphysik-Programm. 
bevorzugte Gegenstand dieses Textes nicht ,Deus‘, sondern ,res‘, und wir können daher vermuten, dass der primäre Gegenstand von Spinozas Metaphysik, anders als es oft behauptet wurde, in der Realität und der ontologischen Bestimmtheit der Einzeldinge liegt, die uns auch im alltäglichen Leben begegnen, und nicht im Gottesbegriff. Letzterer ist zwar für gewisse argumentative Züge wichtig und somit für das resultierende Bild der Realität durchaus relevant. Er steht aber nicht im Zentrum der im ersten Buch der Ethik entwickelten Theorie.

Dass Spinoza in der skizzierten Weise an die Ontologie-Diskussion seiner Zeit anschließt, hat wichtige Implikationen für die Frage, wie weit die von ihm intendierten metaphysischen Korrekturen am Common Sense reichen. Der skizzierte Ausgangspunkt ist zwar mit dem Gedanken kompatibel, dass zahlreiche unserer Alltagsauffassungen, die wir von den Dingen unserer Lebenswelt haben, verworren und dunkel seien und mithin einer Klärung bedürften. Dass es im Zuge einer solchen Klärung zu Korrekturen an unserem Alltagsverständnis der Dinge kommt, ist erwartbar und nicht mit grundsätzlichen Problemen verbunden. Gleichzeitig wird man vor diesem Hintergrund auch annehmen können, dass Spinoza mit seinen Revisionen nur so weit gehen wollte, wie das möglich ist, ohne dass sein eigenes Vorgehen unterminiert würde. Letzteres wäre aber der Fall, wenn entweder dem Gegenstand, von dem wir ausgegangen sind, jegliche unserem Denken und Verstehen vorgängige Existenz abgesprochen würde, oder die Voraussetzungen negiert würden, die eine mentale Fokussierung auf einzelne Dinge möglich machen.

Insgesamt wird man daher die Frage danach, ob wir es bei Spinozas Metaphysik mit einem deskriptiven oder einem revisionären Ansatz zu tun haben, mit einem abwägenden „sowohl als auch“ beantworten müssen. Zum einen ist der Bezugspunkt von Spinozas Metaphysik mit jenem von Strawsons deskriptiver Metaphysik durchaus vergleichbar. Anders als dieser orientiert sich Spinoza zwar nicht an unserer Alltagssprache; gleichwohl setzt er bei denjenigen Dingen an, mit denen wir in unserem alltäglichen Leben zu tun haben. Allerdings sucht er im Zuge seiner Analyse nicht einfach unsere alltäglichen Vorstellungen, die wir von diesen Dingen haben, zu explizieren und zu bestätigen. Im Gegenteil, wir können vermuten, dass er in seiner Metaphysik auf beträchtliche Revisionen an unserem Common Sense aus ist. Auch wenn man daher begründete Zweifel daran hegt, ob Spinoza mit seinem Rationalismus tatsächlich so weit gegangen ist, wie Della Rocca ihn gehen lässt, wird man feststellen müssen, dass Spinoza in manchen Punkten an unserem Alltagsverständnis rüttelt und sich dieses durch eine Auseinandersetzung mit der Ethik womöglich stark verändert. 


\section{5}

Es stellt sich zum Abschluss noch die Frage, ob wir heute mit Della Roccas radikalem Spinoza nicht besser bedient sind als mit dem skizzierten historisch gemilderten Interpretationsversuch. Verhilft uns der idealistische Monismus, auf den der doppelte Gebrauch des PSR hinausläuft, nicht zu einem viel konsequenteren und besser begründeten Verständnis davon, was ist, als der nur maßvoll revisionäre, realistische Rationalismus, den man Spinoza vermutlich attestieren müsste, wenn man auf den historischen Hintergrund Rücksicht nähme?

Wenden wir uns zur Erörterung dieser Frage nochmals den spezifischen Desiderata zu, derer die Metaphysik Rechnung tragen sollte. Soviel scheint klar: Wo sich diese Disziplin nicht darauf beschränkt, die philosophischen Implikationen einzelwissenschaftlicher Theorien zu diskutieren, da steht sie letztlich - in der einen oder anderen Weise - im Dienst unseres menschlichen Selbstverständnisses. Ob man allerdings diesen Dienst in der Explikation und Klärung unseres alltäglichen Begriffssystems ansetzt oder ob man darüber hinaus eine Revision desselben für nötig hält, hängt von weiteren Annahmen ab, so z. B. jener, ob man glaubt, dass Menschen für Leid verursachende Selbstmissverständnisse anfällig sind. Zur Diskussion steht also, kurz gesagt, ob menschliche Selbstverständnisse therapiebedürftig und -fähig sind.

Nun ist die Rede von der Philosophie als einer Therapie eine zweideutige. Zum einen wird der Philosophie manchmal im Anschluss an den späten Wittgenstein eine therapeutische Funktion zugesprochen. Dieser Verwendung der Metapher zufolge liegt das hauptsächliche Ziel unserer Bemühungen in der Auflösung und Heilung von Begriffsverwirrungen, deren Ursprung in einem irreführenden philosophischen Theoretisieren zu suchen sei. Im Zentrum dieses an Wittgenstein orientierten therapeutischen Verständnisses von Philosophie steht somit die Annahme, dass Philosophie keine Theorie ist und sein sollte - eine Annahme, die nicht zuletzt von der deskriptiven Metaphysik geteilt wird. ${ }^{44}$

Zum anderen ist bisweilen auch mit Blick auf gewisse Ansätze der Antike und der frühen Neuzeit von einem therapeutischen Philosophieverständnis die Rede, wobei gerade die Ethik ein Paradebeispiel dafür darstellt. ${ }^{45}$ Rein methodisch gesehen, bestehen durchaus gewisse Ähnlichkeiten zu Wittgenstein. So

44 Auch Strawson rekurriert bei seiner Erörterung der Aufgabe der analytischen Philosophie auf diese Metapher. Obwohl er sich von Wittgenstein abgrenzt und dem Modell der Grammatik den Vorzug gibt, assoziiert er aber die Therapie-Metapher klar mit einem deskriptiven und nicht einem revisionären Metaphysikverständnis; vgl. Strawson (1992), 3-5.

45 Einen Überblick über die frühe Neuzeit gibt Garrett (2012). 
besteht auch Spinoza zufolge das therapeutische Potential der Philosophie in einer Klärung dunkler und verworrener Begriffe oder Ideen. Dass eine solche Klärung nötig ist, liegt ihm zufolge aber nicht an einer falschen Theorieorientierung der Philosophie, sondern an der Tatsache, dass wir uns als endliche, mit Bewusstsein begabte Wesen zwar etlicher Dinge gewahr sind, uns gleichzeitig aber weitgehend im Unwissen darüber befinden, was wir sind und können. Es ist Spinoza zufolge ein wesentlicher Aspekt der menschlichen Existenzbedingungen, dass wir uns für freier halten als wir sind, weswegen wir auch dazu neigen, uns als besondere und außerhalb der Gesetze der Natur stehende Wesen zu begreifen. ${ }^{46}$ Dies schließt nicht aus, dass wir ein besseres Wissen über uns selber erwerben können. Als endliche Wesen sind wir dazu aber zwingend auf Lernprozesse angewiesen, mittels derer wir unsere Ideen adäquater begreifen lernen können. In gewisser Weise ist diese Angewiesenheit auf Lernprozesse für Spinoza ein wesentlicher Ausdruck unserer Endlichkeit.

Ich denke, dass diese Diagnose der Ursachen unserer Therapiebedürftigkeit für die Beurteilung metaphysischer Ansätze ganz entscheidend ist. Spinoza unterstellt hier - völlig zu Recht, wie ich meine - einen Zusammenhang zwischen den menschlichen Existenzbedingungen einerseits und unseren Desiderata an eine Metaphysik andererseits. Folgt man daher Spinozas therapeutischem Philosophieverständnis, so müssen wir Metaphysik als Unterfangen begreifen, das dazu dient, uns bei der Entledigung von unseren Selbstmissverständnissen zu helfen. Letztere sind dabei allerdings nicht einfach die Folge falscher Theorien, sondern haben mit der Tatsache zu tun, dass wir uns aufgrund unserer Existenzbedingungen als endliche Wesen Auffassungen über die Dinge und die Welt zurechtlegen müssen, ohne über hinreichendes Wissen zu verfügen. Dies legt einerseits nahe, dass sich die Metaphysik nicht damit begnügen kann, unser alltägliches Begriffssystem zu explizieren. Andererseits wird vor diesem Hintergrund auch klar, warum wir nicht darum herumkommen, uns mindestens zunächst an gewissen alltäglichen Überzeugungen zu orientieren. Metaphysik ist zwar im besten Fall darauf aus, die Realität sub specie aeternitatis zu repräsentieren, dennoch wird sie von endlichen Wesen betrieben, die auf Lernprozesse angewiesen sind. Wo dies missachtet wird, wird die Stipulation eines göttlichen Gesichtspunkts leicht zur Anmaßung. Dies ist auch das Problem eines metaphysischen Ansatzes, der sich als direkte Auseinandersetzung mit der Realität begreift. Wo nicht mindestens der Ausgangspunkt in der Deskription und Analyse unseres alltäglichen

46 Die einschlägige Passage dazu ist der Anhang zum ersten Buch der Ethik. Vor ihrem Hintergrund hat Louis Althusser seinerzeit in der die französische Rezeption prägenden Stellungnahme von 1972 Spinoza eine Tendenz zur Ideologiekritik attestiert (vgl. Althusser 1998, 184). 
Verständnisses der Dinge liegt, da geht schlicht vergessen, dass Metaphysik von Menschen betrieben wird. Wo sie umgekehrt gänzlich darin aufgeht, da verkennt sie, dass Menschen an ihrem alltäglichen Begriffssystem bisweilen erheblich leiden. Metaphysik, die unseren Desiderata an diese Disziplin gerecht werden will, muss deskriptiv anfangen und revisionär werden. Oder anders gesagt: Wo sie nicht deskriptiv anfängt, ist sie verblendet; wo sie nicht revisionär wird, läuft sie leer. ${ }^{47}$

\section{Literatur}

Althusser, L. (1998), Éléments d'autocritique [1972], in: Solitude de Machiavel, hg. v. Sintomer, Y., Paris, 159-197.

Bennett, J. (1984), A Study of Spinoza's Ethics, Indianapolis.

Brun, G. (2014), Reflective Equilibrium Without Intuitions?, in: Ethical Theory and Moral Practice 17, 237-252.

Curley, E. M. (1969), Spinoza's Metaphysics. An Essay in Interpretation, Cambridge, Mass.

Curley, E., u. Walski, G. (1991), Spinoza's Necessitarianism Reconsidered, in: Gennaro, R., u. Huenemann, C. (Hg.), New Essays on the Rationalists, Oxford, 241-262.

Della Rocca, M. (1996), Representation and the Mind-Body Problem in Spinoza, Oxford.

Della Rocca, M. (2002), Spinoza's Substance Monism, in: Biro, J., u. Koistinen, O. (Hg.), Spinoza: Metaphysical Themes, Oxford, 11-37.

Della Rocca, M. (2003), A Rationalist Manifesto: Spinoza and the Principle of Sufficient Reason, in: Philosophical Topics 31, 75-93.

Della Rocca, M. (2008), Spinoza, New York.

Della Rocca, M. (2012a), Rationalism, Idealism, Monism, and Beyond, in: Förster, E., u. Melamed, Y. (Hg.), Spinoza and German Idealism, Cambridge, 7-26.

Della Rocca, M. (2012b), Violations of the Principle of Sufficient Reason (in Leibniz and Spinoza), in: Correia, F., u. Schnieder, B. (Hg.), Metaphysical Grounding. Understanding the Structure of Reality, Cambridge, 139-164.

Della Rocca, M. (2013), The Taming of Philosophy, in: Laerke, M., Smith, J. E. H., u. Schliesser, E. (Hg.), Philosophy and Its History. Aims and Methods in the Study of Early Modern Philosophy, New York u. Oxford, 178-208.

Elgin, C. Z. (1996), Considered Judgment, Princeton, N. J.

47 Ich danke Georg Brun für seine kritische Lektüre einer früheren Version und die dabei artikulierten kritischen Einwände; Simon Berwert und Robert Schnepf für wichtige Hinweise betreffend verschiedener Details; und den Teilnehmerinnen und Teilnehmern des Konstanzer Fachbereichs-Kolloquium für ihre Diskussionsbereitschaft. Bei Michael Della Rocca schließlich möchte ich mich nicht nur dafür bedanken, dass er mir das Manuskript seiner Whitehead Lectures zur Verfügung gestellt hat, sondern auch für den nie versiegenden philosophischen Austausch über Metaphysik in den vergangenen dreizehn Jahren. 
Garrett, A. (2012), Seventeenth-Century Moral Philosophy. Self-Help, Self-Knowledge, and the Devil's Mountain, in: Crisp, R. (Hg.), Oxford Handbook of the History of Ethics, Oxford, 229-279.

Garrett, D. (1979), Spinoza’s ,Ontological‘ Argument, in: The Philosophical Review 88, 198-223.

Garrett, D. (1991), Spinoza's Necessitarianism, in: Yovel, Y. (Hg.), God and Nature. Spinoza's Metaphysics, Leiden, 191-218.

Goclenius, R. (1964), Lexicon Philosophicum [Frankfurt am Main 1613], Hildesheim.

Kremer, K. (1984), Ontologie, in: Ritter, J., u. Gründer, K. (Hg.), Historisches Wörterbuch der Philosophie 6, Basel, Sp. 1189-1198.

Laerke, M. (2011), Spinoza's Cosmological Argument in the Ethics, in: Journal of the History of Philosophy 49, 439-462.

Laerke, M. (2014), Spinoza et le „PSR“ : représentations, concepts, idées, in: Archives de Philosophie 77, 721-726.

Melamed, Y. (2012), The Sirens of Elea. Rationalism, Monism and Idealism in Spinoza, in: Goff, P. (Hg.), Spinoza on Monism, Basingstoke, 78-89.

Newlands, S. (2010), Another Kind of Spinozistic Monism, in: Noûs 44, 469-502.

Newlands, S. (2012), Thinking, Conceiving, and Idealism in Spinoza, in: Archiv für Geschichte der Philosophie 94, 31-52.

Perler, D. (2006), Das Problem des Nezessitarismus, in: Hampe, M., u. Schnepf, R. (Hg.), Ethik in geometrischer Ordnung dargestellt, Berlin, 59-80.

Perler, D. (2015), Gibt es Individuen? Überlegungen zu Spinozas Monismus, in: Deutsche Zeitschrift für Philosophie 63.3, 497-517 (Beitrag in diesem Heft).

Rawls, J. (1979), Eine Theorie der Gerechtigkeit, übers. v. Vetter, H., Frankfurt am Main.

Renz, U. (2009), Explicable Explainers. The Problem of Mental Dispositions in Spinoza's Ethics, in: Damschen, G., Schnepf, R., u. Stüber, K. R. (Hg.), Debating Dispositions. Issues in Metaphysics, Epistemology and Philosophy of Mind, Berlin, 79-98.

Renz, U. (2010), Die Erklärbarkeit von Erfahrung. Realismus und Subjektivität in Spinozas Theorie des menschlichen Geistes, Frankfurt am Main.

Renz, U. (i. E.), Finite Subjects in the Ethics. Spinoza on Indexical Knowledge, the First Person and the Individuality of Human Minds, in: Della Rocca, M. (Hg.), Oxford Handbook of Spinoza, Oxford.

Schnepf, R. (1996), Metaphysik im ersten Teil der Ethik Spinozas, Würzburg.

Schütt, H.-P. (1985), Spinozas Konzeption der Modalitäten, in: Neue Hefte für Philosophie 24-25, 166-183.

Spinoza, B. de (1999), Ethik in geometrischer Ordnung dargestellt, übers. und hg. v. Bartuschat, W., Hamburg.

Strawson, P. F. (1971), Individuals. An Essay in Descriptive Metaphysics [1959], London u. New York.

Strawson, P. F. (1992), Analysis and Metaphysics. An Introduction to Philosophy, Oxford.

Van Inwagen, P. (2013), Metaphysics, in: Zalta, E. N. (Hg.), The Stanford Encyclopedia of Philosophy (Winter 2013 Edition), URL: http://plato.stanford.edu/archives/win2013/ entries/metaphysics/ (abgerufen am 13.6.2015).

Wilson, M. D. (2001), Ideas and Mechanism. Essays on Early Modern Philosophy, Princeton, N. J. 